\title{
Does the article, "Mass screening for gastric cancer: how to select patients for endoscopic examination," satisfy criteria of scientific quality?
}

\author{
Masao Kobayakawa
}

Received: 14 October 2010/Accepted: 15 December 2010/Published online: 23 February 2011

(C) The International Gastric Cancer Association and The Japanese Gastric Cancer Association 2011

To the Editor:

In the article by Nakashima et al. [1] in a recent issue of Gastric Cancer, gastric cancer detection rates were compared between an X-ray screening group and a gastroenteroscopy (GS) direct group. However, I think there are serious problems of scientific evaluation in that study, and, further, the effectiveness of the initial X-ray screening before endoscopy was not revealed from the study's analysis.

First, the main outcome of the study, the cancer detection rate, should have been compared between the 38,971 subjects who underwent initial X-ray screening and the 5160 subjects who underwent direct GS (0.095 vs. $0.45 \%$, respectively). The gastric cancer detection rate of direct GS was about 4.7 times higher than that of initial X-ray screening followed by GS.

Second, there were no baseline characteristics of the subjects, other than age, for comparing the two groups. As this study was not a randomized controlled trial, it is important to examine whether there were any biases that could have influenced the results, such as age, gender, Helicobacter pylori infection, the screening intervals, or a history of gastric cancer. The reason why only one advanced gastric cancer was detected in the GS direct group might be that subjects in the GS direct group tended to undergo screening more frequently.

If the prevalence of gastric cancer was not different in the two groups, more than $78 \%$ of gastric cancers detectable by GS direct examination could have been missed by $\mathrm{X}$-ray screening followed by endoscopy.

\section{Reference}

1. Nakashima H, Nagahama R, Yamamoto T, Ohkura Y. Mass screening for gastric cancer: how to select patients for endoscopic examination. Gastric Cancer. 2010;13:78-83.

Article related object doi:10.1007/s10120-011-0029-1.

M. Kobayakawa ( $\square)$

Department of Gastroenterology, National Center for Global

Health and Medicine Hospital, 1-21-1 Toyama,

Shinjuku-ku, Tokyo 162-8655, Japan

e-mail: mkobaya@hosp.ncgm.go.jp 